\title{
La atenuación en Barranquilla: estudio sociopragmático*
}

\author{
YASMÍN TORRES FONTALVO** \\ Universidad del Atlántico \\ yasmintorres@mail.uniatlantico.edu.co \\ YOLANDA RODRÍGUEZ CADENA*** \\ Universidad del Atlántico \\ yolandarodriguez@mail.uniatlantico.edu.co
}

Recepción: 06 de diciembre de 2016.

Aprobación: 28 de febrero de 2017.

Forma de citar este artículo: Torres Fontalvo, Y., \& Rodríguez Cadena, Y. (2017). La atenuación en Barranquilla: estudio sociopragmático. Cuadernos de Lingüística Hispánica, (30), 55-79. https://doi.org/10.19053/0121053X.n30.0.6188.

\footnotetext{
* $\quad$ Este artículo es producto del proyecto La atenuación pragmática en el español hablado: su variación diafásica y diatópica dirigido por Marta Albelda y aprobado en la convocatoria de ayuda a proyectos ID "Excelencia” (España) (código FF 12013-40905P).

** Magíster en lingüística, Universidad del Atlántico.

*** Doctora en lingüística, El Colegio de México. Docente Universidad del Atlántico.
} 


\section{Resumen}

En este artículo se analizan los atenuantes que se utilizan en Barraquilla y su relación con variables sociales y pragmáticas, sexo, edad, nivel de instrucción de los hablantes; y el tipo de interlocutor, en sus rasgos, edad y sexo. El análisis tuvo dos etapas: la cualitativa en la que se analizaron las estrategias de atenuación, las marcas y sus funciones pragmático-semánticas asociadas a las funciones y contenidos que los hablantes construyen en sus discursos. La segunda etapa es la cuantitativa que se llevó a cabo con estadística descriptiva e inferencial mediante el software Goldvarb 2001. La muestra se tomó del corpus Preseea que contiene 72 informantes. Los resultados evidenciaron que los atenuantes en Barranquilla covarían con los factores sociales, el género discursivo y el factor pragmático, tipo de interlocutor; este, a su vez, influye en el uso de los atenuadores, cuando el hablante y el interlocutor coinciden en el sexo.

Palabras clave: estudio sociopragmático, atenuación, comunidad de habla, variables sociolingüísticas.

\section{Linguistic Attenuation in Barranquilla: a Sociopragmatic Study}

\section{Abstract}

This article analyzes some of the most frequent attenuating factors used by native Spanish speakers from Barranquilla, while taking into consideration the relation of these factors with social and pragmatic variables such as sex, age, and level of education of the interlocutor. It was a two - stage analysis: the first stage was a qualitative study of the attenuating strategies, markers, pragmatic and semantic functions used by the speakers in their speeches. The second stage was the quantitative phase of research, which developed descriptive and inferential statistics, using the Goldvarb 2001 software. The research sample was taken from the Preseea corpus, which contains 72 informants. The results showed that attenuating factors in Barranquilla vary in accordance with social factors, discursive genres and pragmatic aspects. The use of attenuating factors is also influenced by the type and sex of the interlocutor.

Key words: sociopragmatic study, attenuation, speech community, sociolinguistic variables. 


\section{Latténuation à Barranquilla: étude sociopragmatique}

\section{Résume}

Dans cet article on analyse les atténuants qui sont utilisés à Barranquilla et leur rapport avec des variables sociales et pragmatiques, sexe, âge, niveau de formation du locuteur, et le type d'interlocuteur dans ses traits, âge et sexe. L'analyse a eu deux étapes: la qualitative, dans laquelle on a analysé les stratégies d'atténuation, les marques et leurs fonctions pragma-sémantiques associées aux fonctions et aux contenus que les locuteurs construisent dans leurs discours. La deuxième étape es la qualitative, qui a eu lieu avec un statistique descriptive et inférentielle, en utilisant le software Goldvarb 2001. L'échantillon a été pris du corpus Preseea, composé de 72 informateurs. Les résultats ont montré que les atténuants à Barranquilla covarient avec les facteurs sociaux, le genre discursif et le facteur pragmatique, et le type d'interlocuteur. Celui-ci a une influence sur l'utilisation des atténuants, quand le locuteur et l'interlocuteur ont le même sexe.

Mots clés: étude sociopragmatique, atténuation, communauté de langue, variables sociolinguistiques.

\section{Atenuação em Barranquilla: Estudo sociopragmatico}

\section{Resumo}

Este artigo analisa os atenuadores que são utilizados em Barranquilla e sua relação com variáveis sociais e pragmáticas, sexo, idade, nível de instrução dos falantes; E 0 tipo de interlocutor, nas suas características, idade e sexo. A análise teve dois estágios: 0 qualitativo em que as estratégias de atenuação, as marcas e suas funções pragmáticas-semânticas associadas às funções e conteúdos que os falantes construíram em seus discursos foram analisadas. 0 segundo estágio é o quantitativo que foi realizado com estatística descritiva e inferencial usando o software Goldvarb 2001. A amostra foi retirada do corpus Preseea contendo 72 informantes. Os resultados mostraram que os atenuadores em Barranquilla covariam com os fatores sociais, o gênero discursivo e o fator pragmático, tipo de interlocutor; isso, por sua vez, influencia 0 uso dos atenuadores, quando o interlocutor e 0 interlocutor concordam com o sexo.

Palavras-chave: estudo sociopragmático, atenuação, comunidade de fala, variáveis sociolinguísticas. 


\section{Introducción}

El presente estudio es una investigación sociopragmática cualitativa con enfoque variacionista en sus resultados cuantitativos, que analiza los atenuadores en la comunidad de habla barranquillera. Se han seleccionado variables independientes de tipo social, como la edad, el sexo y el nivel educativo; de tipo pragmático, la clase de interlocutor; y la variable independiente de tipo discursivo correspondiente al género. El corpus analizado es el Preseea-Barranquilla, constituido por 72 conversaciones semidirigidas de informantes preestratificados. Los resultados demuestran que sí hay una influencia de estas variables en la frecuencia y probabilidad de uso de los atenuadores en la comunidad elegida; y esto se relaciona con estudios fuera de Colombia.

\section{Aspectos teóricos}

Dados los objetivos de esta investigación, se plantean los conceptos teóricos que la sustentan: comunidad de habla, atenuación, cortesía verbal y géneros discursivos.

La comunidad de habla está formada por un grupo de hablantes que comparte al menos una variedad lingüística, unas reglas de interpretación de uso, unas actitudes y unas mismas valoraciones de las formas lingüísticas (Gumperz, 1971; Labov, 1966; Hymes, 1974; Romaine, 1982; Moreno Fernández, 1977; Silva Corvalán, 2001). En este sentido, Barranquilla es una comunidad de habla, teniendo en cuenta que sus habitantes "conviven en una misma área geográfica, poseen un perfil sociocultural formado a través de procesos históricos, comparten una competencia lingüística (el español), un mismo dialecto (español del Caribe) y modos de interacción lingüística y social" (Rodríguez Cadena, 2009, p. 69).

El segundo concepto clave en la presente investigación es el de atenuación, que se define como una categoría pragmática cuya función consiste en minimizar la fuerza ilocutiva de los actos de habla, y con frecuencia regula la relación interpersonal y social entre los participantes de la enunciación; el hablante la usa para proteger su propia imagen positiva y la del interlocutor. Además, reduce el valor significativo de un enunciado (Briz, 2003; 2007; Haverkate, 1994; Puga, 1997; Ballesteros 2002; Albelda, 2005, 2009). 
La atenuación ${ }^{1}$ en Barranquilla se relaciona en ocasiones con la cortesía verbal, la cual, desde el punto de vista discursivo, se propone lograr la colaboración tanto del hablante como del oyente y evitar las tensiones de la conversación. La cortesía verbal utiliza la atenuación como estrategia para proteger al interlocutor de posibles ofensas o heridas. Generalmente, la cortesía mitigadora se realiza mediante procedimientos de atenuación lingüística. Algunos autores proponen dos tipos de cortesía: una cortesía mitigadora de carácter negativo que trata de evitar o reparar una posible amenaza al oyente; y una cortesía valorizante de carácter positivo, que pretende producir actos corteses, obviando el posible riesgo de amenaza al oyente (Goffman, 1959, 1967; Kerbrat y Orecchioni, 1996; Brown y Levinson, 1987).

El último concepto que es necesario definir en esta investigación, es el de género discursivo, por cuanto en Barranquilla, incide en la variabilidad de los recursos de atenuación que usa la comunidad de habla. En el presente estudio solo se abordaron los modos narrativo y argumentativo, para cuya identificación en las muestras de análisis seleccionadas, se tomaron los tipos de estructura y elementos que los caracteriza. En el primero, se asume la estructura de la narrativa cotidiana (Labov, 1972) formada por: el resumen, en el cual el hablante-narrador hace una síntesis de lo que va a contar; la orientación, en la que se dan datos temporales, espaciales e informativos sobre los personajes 0 actores; la evaluación, que apunta a los juicios que el hablante hace sobre lo que está contando con el fin de demostrar lo interesante de su relato; la acción complicante en la que se da el clímax de la narración; la resolución donde se narra cómo se desenvolvió el asunto; y la coda, que actúa como cierre del relato.

En cuanto a la estructura argumentativa, para seleccionar las muestras de análisis, se tomaron los elementos obligatorios y opcionales, los cuales son: una posición 0 punto de vista (P): se reconoce porque en él el hablante expone su convicción o posición frente a un hecho o situación; un Fundamento (F): son las razones que justifican el punto de vista de tal manera que posea confiabilidad y pueda ser aceptado por los oyentes; un Garante (G): se refiere al principio explícito o implícito que está entre el punto de vista (P) y el fundamento que la sustenta (F). Y los elementos opcionales: un condicionamiento del

1 El tema de la atenuación se desarrolló en la bibliografía tanto teórica como empíricamente, mediante el estudio de ciertas marcas (Lotfi A. Zadeh y Uriel Weinreich, 1965, 1966; Lakoff, 1973); y luego, bajo el término "mitigation" (Brown and Levinson, 1987, Caffi, 1999, Fraser, 1999, Martinovski, 2000, Von Eemeren and Grootendorst, 2004). En las investigaciones hispanas se encuentran los autores que iniciaron en España (Puga, 1997, Briz, 2001, 2002; Bravo y Briz, 2004; Garrido, 2006; Hidalgo, 2006; Albelda, 2004, 2005, 2013). En Colombia se registran artículos sobre el tema (Cisneros, 2007; Coutin, 2010). En Barranquilla el primer trabajo sobre el tema es la ponencia de Rodríguez Cadena (XV COLOQUIO DE EDICE, 2010); y la tesis de maestría de Torres Fontalvo (2014), en el que se realiza un estudio exhaustivo sobre los mecanismos atenuadores y los factores sociales y pragmáticos que influyen en sus usos, funciones y distribución en la comunidad de habla barranquillera. 
punto de vista (Cd) que se usa para impedir previamente los argumentos que invaliden el punto de vista; una concesión (C) en la que se cita un punto de vista que se opone al del hablante que argumenta; y una refutación (R) que responde a la concesión de manera anticipada y sirve para rebatir lo que se espera contradiga el punto de vista del hablante (Charaudeau, 2001; Martínez Solís; 2002).

\section{Materiales y métodos}

El análisis se basa en el corpus Preseea-Barranquilla, que contiene 72 entrevistas semidirigidas o conversaciones semidirigidas, recolectadas en los entornos cotidianos de los hablantes. Se tomaron los primeros 10 minutos de las entrevistas. Las variables independientes son de dos tipos: externas, sociales y pragmáticas; las primeras son: sexo (hombre, mujer), nivel de instrucción (primaria, bachillerato y universidad), edad (20-34 años, 35-54 años y 55 en adelante), y el tipo de interlocutor; y las internas están constituidas por los géneros discursivos. La variable dependiente son las marcas de atenuación y sus tipos.

El proceso de análisis tuvo dos etapas: la cualitativa en la que se analizaron las estrategias de atenuación de los barranquilleros, las marcas y sus funciones pragmático-semánticas asociadas a las funciones y contenidos que los hablantes construyen en sus discursos. La segunda etapa es la cuantitativa que se llevó a cabo con estadística descriptiva e inferencial; esta se realizó mediante el software Goldvarb 2001.

\section{Resultados}

\subsection{Análisis cualitativo: los atenuantes en Barranquilla}

La comunidad de habla barranquillera usa diversos recursos para la atenuación: categorías gramaticales, expresiones y formas léxicas, pasan a cumplir un rol discursivo de mitigación. Para el análisis cualitativo y cuantitativo, se tuvo en cuenta la ficha del proyecto Es.Por.Atenuación (2013), en la que se proponen los siguientes criterios para establecer el carácter o función atenuadora de una categoría o expresión lingüística, entre otros: (a) definir el contexto general: participantes, características sociales, estatus, roles; (b) definir la situación comunicativa y el contexto interactivo concreto; (c) definir el contexto anterior y posterior en que se integra. Con base en esto, se trata de determinar: cuál es el desencadenante, el segmento atenuado, el atenuante y, en ocasiones, el efecto que podría causar la atenuación en el interlocutor.

Cabe preguntarse, ¿cuál es la función de la atenuación en la conversación semi-informal de los hablantes barranquilleros? El análisis de los datos lleva a plantear 
que ella cumple la función estratégica que va más allá de la simple cortesía, esto es, el YO tiene la intención de lograr sus propios intereses; siguiendo a Briz (2001), es una estrategia de discurso que "más que deferencia auténtica hacia el interlocutor, persigue... el propio interés del hablante" (p. 110).

\section{LA ATENUACIÓN Y LOS ACTOS DE HABLA EN BARRANQUILLA}

En Barranquilla, los hablantes usan los atenuadores en diferentes proporciones, dependiendo de los actos de habla que se lleven a cabo; según estos, los hablantes pueden emplear un atenuador o varios conforme al grado de mitigación que desee construir con el objetivo de guardar su propia imagen y no comprometerse con el contenido de lo que está enunciando. Albelda (2006) ya ha anotado esta relación entre el uso de atenuadores y actos comunicativos; se tomó esta propuesta de actos de habla: 1. Recriminación. 2. Desafíos (encararse al interlocutor), desconfianza (muestras de recelo y de duda sobre el interlocutor). 3. Peticiones (y órdenes) en beneficio del hablante. 4. Peticiones (y órdenes) en beneficio del oyente. 5. Comentarios evaluativos negativos y calificaciones peyorativas sobre la persona 0 las acciones del interlocutor. 6. Insultos y comentarios despreciativos directos a la imagen de los interlocutores. 7. Consejos. 8. Negar algo al interlocutor. 9. Contradecir lo dicho a la opinión expresada por el interlocutor. 10. Quejas sobre el interlocutor. 11. Corregir lo dicho por el interlocutor. 12. Prohibiciones. Con base en esta clasificación, se estudiará lo que ocurre en Barranquilla:

\section{Atenuador: vacilación por alargamiento y repetición; uso de diminutivo:}

ENTREVISTA 49. Nivel bajo. Mujer de 21 años.

A: pro (palabra cortada) / ahora es que vamos a salir íbamos a ir a ver un:

// un carro de perro que están vendiendo / o sea un carrito / a eso íbamos.

B: yo creo que

En la entrevista se mitiga el enunciado con el fin de dar la imagen de humildad hacia el interlocutor. Se aprecia que la estrategia atenuadora se dispara con el alargamiento vocálico y la repetición, para luego consumarse en el uso del diminutivo. Este proceso se ve con mucha frecuencia en las conversaciones semidirigidas de la comunidad de habla barranquillera; se podría plantear que los hablantes crean un marco de la atenuación, con un inicio en el que usan una estrategia atenuadora, para después incrementar la estrategia atenuadora y consumarla plenamente; este incremento depende del acto de habla que se construye; he aquí otro ejemplo en el que se utilizan varios recursos atenuadores: 
Atenuadores: vacilación por alargamiento y repetición, marcador retardador, explicación, cuantificador, uso del "como" atenuador, uso del "no saber":

Entrevista 1. Joven de 21 años, mujer, enfermera. Nivel alto

\begin{tabular}{|c|c|}
\hline CONTEXTO ANTERIOR: & ATENUACIÓN \\
\hline $\begin{array}{l}\text { A: (simultáneo) y es que eso está por dentro } \\
\text { (simultáneo) / es que la gente / } \\
\text { bueno (pu) pues los hombres le tiene miedo } \\
\text { a las mujeres / y también los / } \\
\text { las (vacilación) mujeres le tienen miedo a } \\
\text { esos hombres así que andan: / son } \\
\text { promiscuos / que las mujeres son promi:s- } \\
\text { cuas / entonces le tiene miedo } \\
\text { entonces / dicen por / «(a:) como ella es } \\
\text { promiscua / ella se acuesta con el } \\
\text { que venga / pues ella puede tener VIH / tam- } \\
\text { bién puede tener otra } \\
\text { enfermedad de tipo»/ pero eso es //. } \\
\text { B: ¿es relativo? }\end{array}$ & $\begin{array}{l}265 \text { A: sí es relativo / las jovenci:tas de hoy en } \\
\text { día / hay chicas / que se han muerto } \\
\text { con la misma edad mía / se han muerto } \\
\text { de menos edad / ipor qué? porque } \\
\text { ya vienen chicos // que: / o sea no saben: } 0: \\
\text { sí saben / entonces hay unos } \\
\text { que lo hacen es como por: / por (vacila- } \\
\text { ción) vengar:se no sé: / porque esa } \\
\text { enfermedad también tiene ese proceso. }\end{array}$ \\
\hline
\end{tabular}

Contexto: tema: muerte; acto de habla: acusación. En este discurso se observa que el tema es comprometedor, porque el informante está hablando de la muerte de alguien que tiene sida; se encierra aquí una acusación posible y se trata de mitigar con varios recursos de atenuación: las vacilaciones por alargamientos vocálicos y repetición, el marcador discursivo "o sea" que cumple la función de aclaración introductoria de un enunciado mitigado "no saben"; pero se aprecia que inmediatamente la informante cambia su afirmación usando la aseveración "sí saben", que está antecedida por la mitigación dada en la vacilación con el alargamiento. El hablante se da cuenta del contexto amenazante; por lo tanto, regresa a la atenuación y aumenta los recursos: con la delimitación "hay unos", el uso de "ser" focalizador "lo hacen es como por", que, al plantear la construcción seudohendida, también actúa como retardador; el "como" atenuador, la vacilación de la preposición "por" y el uso del "no saber".

A continuación, se ofrece otro ejemplo de este mismo hablante:

A: no: / me dicen negra / pues / cuando me dicen negra // yo: ve (palabra cortada) enseguida / miro / pues la mayoría a mí me dicen negra / me dicen morena / me dicen Siris* / pero más me dicen negra y Siris* // (pore) porque yo digo / bueno mi color / a mí encan:ta / cuando ya se ponen que y que: «esa negra» / cuando ya se ponen con: / como con: (vacilación) / 
discriminación y así que / «ay èy esa negra qué?» / entonces «ique esa negra qué te pasa?» / «este es el color / más lindo y más firme que hay mamita / tu eres linda ahora / pero cuando tú estés viejas / nos vamos a comparar para ver / cuál de las dos es más bonita» / porque / la piel de ustedes / me lo perdona / la piel de ustedes se pone como / más flácida / y se arruga con más rapidez / en cambio que la piel negra no se arruga con rapidez / dura más para arrugarse.

Contexto: tema: discriminación racial; acto de habla: acusación. La hablante empieza a tratar un tema álgido sobre la discriminación; nótese que es el mismo contexto de la muestra discursiva anterior en el que se hace una acusación (que hay discriminación racial en Barranquilla). Por ello, la hablante, cuando llega al punto de usar la denominación, empieza a desplegar estrategias atenuadoras como: la vacilación "con: / como con:" y el "como" atenuador. Se aprecian dos contextos discursivos: (a) el discurso citado en el que la hablante está contendiendo con la persona que le dice "negra" como ofensa; y allí no hay estrategias atenuadoras; (b) el discurso directo con intensificaciones: el uso del demostrativo "este" y el artículo "el", los comparativos "más", reiterados; el uso del adjetivo fuerte "vieja". Pero cuando la hablante deja de citar, y pasa a referirse al entrevistador cuya piel es clara, emplea la atenuación para minimizar la amenaza hacia el oyente, utilizando la expresión de disculpa "me perdona" y el "como" atenuador: "como / más flácida". La hablante previene una posible amenaza a la imagen del otro, hay salvaguarda yo-tú; previene lo que puede ofender, atacar o dañar la imagen del otro.

El siguiente ejemplo ilustra el contexto de acusación donde emergen los atenuadores:

(El entrevistador pregunta ¿Cómo es la relación con su papá?)

A: ¿con mi papito? / la relación / es / escasa / porque él / es un señor / de poco hablar // yo también soy una chica de poco hablar / él tiene / un temperamento todo raro / que él no se relaciona mucho mucho (repetición de palabra) así con las personas no (silencio 3').

A: (simultáneo) es que él es (simultáneo) así: / él es así / o sea / él es así con el de la calle / con el particular por decir / pero ya con aquí el de la casa él no es así / no sé porque / o sea él es un señor / que cuando yo estaba pequeñita yo me acuerdo / yo nunca corría a los brazos de él y que «iay! ahí viene mi papá» no: / porque él era un señor que con esa / como él tiene es (palabra cortada) / bueno / como él / si él lo tiene / esa cara así toda / como agria / cuando él sonríe / se cambia / enseguida / pero ya cuando está serio le preguntan / «iestás rabioso?» / «no»/ él no está rabioso / sino que esa cara toda agria uno niñito ya / uno respeta eso / porque infundía miedo // iya ves?

Se aprecia el contexto de acusación en la valoración negativa del papá; pero la hija no desea calificarlo mal delante del entrevistador; por ello, cuando se acerca la evaluación negativa, la hablante usa las estrategias de atenuación: las explicaciones con alarga- 
mientos, la vacilación, el uso del "no saber", la palabra cortada, el marcador retardador "bueno", el uso del "así" y el "como" atenuador, el pronombre de distanciamiento "uno". Véase cómo la hablante sigue caracterizando a su papá:

A: pero eso fue por él mismo porque / o sea cuando uno está (pelaito) peladito uno las cosas las aprende desde chiquito iverdad? / cuando uno está chiquito / por decir el papá / «venga que voy a revisar las tareas» / que si «iqué está haciendo?» / que si «iqué? ipara dónde va?» na:da / sino que cuando iba decir una: / una (vacilación) cosita ahí no (risa) / era con una así / con una (vacilación) voz y él tiene un tono de voz gruesa / y él habla como con fuer:za / entonces ya / cuando él le habla a una persona con fuerza / entonces ya esa persona coge miedo por decir / ya yo cojo miedo / yo digo «ah no / yo a eso no le digo más nada» / porque imagínese.

Nuevamente, el contexto de la evaluación negativa del padre causa el uso de estrategias atenuadoras; cuando se acerca la valoración, se activan las siguientes estrategias: vacilación "una: / una", el uso del diminutivo "cosita", el uso del "ahí", la risa, el uso del "así", nuevamente la vacilación "con una / con una", el "como" atenuador; y el uso de la forma de distanciamiento "esa persona".

En este ejemplo, se observa el acto de habla evaluativo y la aparición de atenuadores:

Entrevista 2 nivel alto.

(Contexto): personalización del discurso, apropiación mediante el uso del "yo".

A: o sea aquí: / hay gente / son negros / pero no tienen conciencia / porque yo por el hecho de ser negro / si yo veo a alguien / que se lanza a ser / que es negro / y se lanza a algo yo voto por él / porque yo soy negra / y yo voto / pero hay muchos que no / hay muchos que votan porque le den diez mil pesos / porque le den esto / porque le den otro / y no por por (vacilación) su compañero / por su amigo / porque surja ino?

Véase ahora cómo después de este contexto, el hablante pasa a atenuar: A: es que no sé / yo digo que es como falta de: / de: (vacilación) / (conciensitación) concientización / después que tú no te hayas concientizado / tú no: / porque si si (vacilación) tú sabes que nosotros fuimos esclavos / que: / nosotros estamos luchando por abolir la esclavitud porque / se haya abolido pero todavía no se ha abolido totalmente / porque tú vas a con (palabra cortada) buscar un trabajo / y como tú eres negra / no te cogen / muchas veces / y en otras partes sí / hay otros que sí / hay otros que no / entonces hay que luchar por eso / hay otra gente que no tiene conciencia en eso / pues si tú: / si ves / los políticos / los políticos te dicen / te voy a dar diez mil pesos / tú le regalas el voto / y ellos no te voltean a ver más / en cambio si tú se lo regalas a un negro / hay posibilidad de que ese negro / salga adelante / sal 
(palabra cortada) y te pueda ayudar a ti / y a tu comunidad.

Aquí, el "como" atenuador se disipa en la evaluación directa contra la falta de concientización en algunos de la comunidad negra. Obsérvese el turno:

A: o sea hay muchos grupos / de comunidades negras / pero la gente / como que no le da: / como la mayor importancia ((ruido)) / aunque / los jóvenes sí: / ya lo están haciendo / pues las personas adultas no lo hacen / solo los jóvenes son lo que lo están haciendo / que le están dando más conciencia.

Aquí, el hablante usa dos "como" atenuadores, tratando de mitigar la intervención fuerte anterior; quizá se dio cuenta de la fuerza de su evaluación negativa; el contexto mitigador se refuerza con los enunciados concesivos que plantean cómo los jóvenes sí toman conciencia. La afirmación inicial con la expresión "la gente", que generaliza la acusación, y que plantearía un contexto amenazante, luego se ve limitada con la separación de los dos grupos, jóvenes y adultos; esto mitiga aún más.

Para concluir este análisis cualitativo, en Barranquilla se utilizan tres estrategias:

(a) La presencia de un atenuador en el enunciado.

(b) La presencia de muchos atenuadores que crean un verdadero marco mitigador relacionado con actos de habla amenazantes: acusaciones, evaluaciones negativas; manejo de temas comprometedores o considerados tabú: "Que entre (risa) comillas pues / de pronto muy adicto así como / adicto al sexo". En este ejemplo se encuentran seis marcadores de atenuación: la expresión "entre comillas", la risa, el marcador retardador "pues", la expresión de duda "de pronto", el uso del marcador "así" y el "como" atenuador. El hablante busca autoprotegerse de lo dicho, salvaguarda su "yo", con el interés de no perder imagen. Esta estrategia de acumulación de atenuadores es muy común en la conversación semiinformal. Véase otro ejemplo: "Digamos que // eso es como relativo / no sé / la verdad era que tendría yo que vivir allá para poder:". El hablante evade la respuesta que lo puede comprometer cuando se le pregunta por una valoración de un barrio peligroso de Barranquilla. Aquí, se aprecian 8 marcadores de atenuación: el uso de la expresión de citación en plural que involucra un "nosotros", el "como" atenuador, el adjetivo mitigador "relativo", el uso de "no saber", el uso del marcador discursivo "la verdad" que refuerza su opinión mitigada; el uso del tiempo pasado imperfecto en el verbo "era" que aleja en el tiempo lo dicho; el uso del condicional que distancia aún más el compromiso; y el alargamiento vocálico. 
(c) La alternancia atenuación-intensificación en la cual pareciera ocultarse el objetivo mitigador tras el contexto intensificador, pero se impone dicho objetivo mediante la construcción semántico-pragmática en el entorno conversacional.

\section{LA ATENUACIÓN Y LOS GÉNEROS DISCURSIVOS EN BARRANQUILLA}

En cuanto a los géneros discursivos, se observa que la atenuación es escasa en la narrativa cotidiana como se corroborará más adelante; en su lugar, prima la intensificación ${ }^{2}$; mientras en el discurso argumentativo y descriptivo, abundan los atenuadores en los diferentes actos de habla que implican amenaza de la imagen, como se ha ilustrado anteriormente en el discurso argumentativo; véanse ahora ejemplos del narrativo.

En la narrativa, baja la frecuencia de atenuadores, y cuando aparecen, lo hacen en el discurso citado con estructuras argumentativas y descriptivas. Esto se explica, en el caso de la narración, porque se autoconstruye la imagen del héroe en el transcurso de los eventos con lo cual se busca impactar al oyente, en especial en la acción complicante; y los otros apartados, la evaluación y la orientación, actúan en relación con esta, por lo tanto, contribuyen al efecto intensificador de las acciones que desea causar el narrador en el oyente.

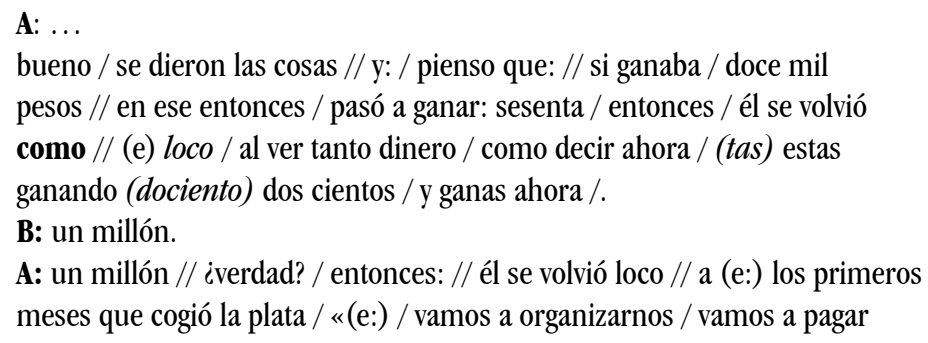

El primer "como" pareciera que tuviera valor comparativo, pero en el siguiente turno, la hablante retoma la intervención y ya no usa el "como". Para poder ver el valor atenuador aquí, es necesario situarse en toda la narrativa. La hablante empieza su narración conversando sobre cómo la abandonó su esposo, con todo lo que ocurrió. En este relato alterna la construcción de dos imágenes: una negativa y otra positiva. En estos ejemplos del "como", se observan los dos valores. Esta interpretación se sustenta en la continuación del turno en el que la informante habla de los amigos del esposo:

2 Esto se corrobora en la tesis de maestría de Jacqueline Cruz Vega, Estudio Sociolingüístico de la intensificación en el Habla de Barranquilla, Universidad del Atlántico, Barranquilla, 2014. 


\begin{abstract}
A: ...
// y él recibió / en una navidad / mucha plata (lapso 2') y: entonces:

/ e:scogió sus amigos / de parranda / escogió los lugares donde tenía que ir:

/ y: llegó un momento en que dijo «no / usted no va / usted se queda cuidando los ni:ños / usted: / se queda aquí porque: / la mujer es para la casa» / entonces andaba con amigos que // no voy a decir que eran malos no // pero que también estaban en la misma tónica de divertir:se / pero ellos estaban en una tónica de divertir:se / tener mujeres / pero guardar sus hoga:res / responder por sus espo:sas / y: bueno no sé qué le paso él / (e:) / empezó a cambiar / fue porque se enamoró // y empezó a cambiar / entonces ya el dinero no se veía // ya el dinero: escaseaba / ya habían más deudas / y entre más se ganaba / más compromiso se hacía ((ruido)) / y: / llegó un momento / en que: (silencio 3’) la: situación se puso muy tensa / porque llegó / a un extremo de perderse / viernes / sábado / domingo / y si el lunes era festivo / también se perdía el lunes festivo /
\end{abstract}

En la conversación semiinformal en Barranquilla, la atenuación surge en la narrativa cotidiana en la parte de la orientación que encierra un discurso descriptivo (se describen lugares, personajes, etc.). Por lo tanto, no se trata exactamente del discurso narrativo en sí, sino de una estructura descriptiva incrustada que está evidentemente relacionada con el relato en su estructura, pero no en la esencia de la secuencialidad de los eventos.

En el ejemplo citado, la hablante pareciera que no quisiera evaluar negativamente y de manera directa a los amigos del esposo, por lo tanto, acude al uso de la negación atenuadora y reiterada (doble negación): "no voy a decir que eran malos no". Luego pasa a contrarrestar lo dicho: "pero que también estaban en la misma tónica de divertir:se / pero ellos estaban en una tónica de divertir:se / tener mujeres / pero guardar sus hoga:res / responder por sus espo:sas”, lo que aparentemente implicaría una evaluación negativa hacia dichos personajes, lo cual eliminaría la atenuación. No obstante, si se toma el turno completo de la informante, se puede observar que dicha evaluación negativa, amenazante, se convierte en positiva cuando compara el comportamiento de estos amigos con el de su esposo quien también quería divertirse y tener mujeres, pero mientras sus amigos no abandonaron su hogar, este sí lo hizo. De tal manera que, el marco mitigador continúa con referencia a los amigos, y actúa como estrategia para intensificar el señalamiento hacia el esposo desertor. Es interesante ver que la atenuación con el enunciado negativo, realmente no apunta a evitar el daño a la imagen de los amigos, sino a crear un punto de comparación en el que el esposo definitivamente es evaluado como el malo, contrario a sus amigos. 
Esto se corrobora cuando la hablante dice:

A:...

y: bueno no sé qué le paso él / (e:)

/ empezó a cambiar / fue porque se enamoró // y empezó a cambiar /

entonces ya el dinero no se veía // ya el dinero: escaseaba / ya habían más

deudas / y entre más se ganaba / más compromiso se hacía ((ruido)) / y: /

llegó un momento / en que: (silencio 3’) la: situación se puso muy tensa /

porque llegó / a un extremo de perderse / viernes / sábado / domingo / y

si el lunes era festivo / también se perdía el lunes festivo

Aquí se ve la alternancia entre atenuación y evaluación negativa, triunfando esta finalmente en toda la narrativa; puesto que la hablante atenúa usando el alargamiento, el marcador "bueno", el uso del "no sé", la vacilación (e:). Sin embargo, pasa a usar un discurso directo y bastante intensificado, construyendo una imagen negativa del esposo desertor; el enunciado "fue porque se enamoró", destruye la atenuación previa. Se aprecian las intensificaciones con los verbos: "no se veía", "escaseaba", "más deudas", los comparativos "entre más...más", el uso del silencio de suspenso, la calificación de la situación "muy tensa", la evaluación negativa "llegó a un extremo" y la enumeración de los días "viernes / sábado / domingo / y...". La intensificación se incrementa cuando la hablante ilustra lo que dice con una de las "perdidas" de su esposo:

A:...

el: primer día que se perdió: / bueno yo lo busqué / por todas las carrete:ras / por todos

los mon:tes / con la policía / pensando que era un hombre muer:to / que lo

habían a matado / y al día / después a los tres días apareció rién:dose / que no: / que él estaba donde un ami:go / entonces ya llega uno a tomar una

actitud de / como de indiferencia / de:

La alternancia de la construcción de la imagen negativa con la estrategia de la intensificación y la construcción de la imagen positiva, mediante la estrategia de la atenuación, es frecuente en la narrativa que se está analizando. En general, es muy escasa la atenuación en la narrativa. Se pueden observar entre dos o tres en promedio, en un discurso completo de este género. Una posible explicación de esto es que en ella se da una construcción del "yo"; el hablante edifica su identidad ante el oyente: "While telling their stories autobiographical narrators often enact a characteristic type of self, and through such performances they can become that type of self" (Worthman, citado en Guerrero, 2011, p. 97). 


\subsection{Análisis cuantitativo}

\subsubsection{Análisis de los tipos de atenuantes}

El análisis cuantitativo permitió determinar cuáles eran los atenuantes más frecuentes en la comunidad de habla barranquillera; véanse los siguientes datos: Diminutivo (d): 12 \%; Explicación: (e): 7 \%; Marcador así: (a): 5 \%; Expresión de duda: (x): 6 \%; Marcadores retardadores: (m): $16 \%$; No saber: (n): $2 \%$; Pronombres de distanciamiento: (p): $24 \%$; Como atenuador: (c): $11 \%$; Verbo de opinión: (o): $11 \%$.

Tabla 1. Atenuantes más frecuentes en la comunidad de habla de Barranquilla

\begin{tabular}{|c|c|c|c|c|c|c|c|c|c|c|c|c|c|c|c|c|c|c|c|c|}
\hline & \multicolumn{2}{|c|}{ d } & \multicolumn{2}{|c|}{ e } & \multicolumn{2}{|c|}{$a$} & \multicolumn{2}{|c|}{$\mathrm{x}$} & \multicolumn{2}{|c|}{ M } & \multicolumn{2}{|c|}{$\mathrm{n}$} & \multicolumn{2}{|c|}{$p$} & \multicolumn{2}{|c|}{$\mathrm{c}$} & \multicolumn{2}{|c|}{0} & \multicolumn{2}{|c|}{ TOTAL } \\
\hline & $\mathbf{N}$ & $\%$ & $\mathbf{N}$ & $\%$ & $\mathbf{N}$ & $\%$ & $\mathbf{N}$ & $\%$ & $\mathbf{N}$ & $\%$ & $\mathbf{N}$ & $\%$ & $\mathbf{N}$ & $\%$ & $\mathbf{N}$ & $\%$ & $\mathbf{N}$ & $\%$ & $\mathbf{N}$ & $\%$ \\
\hline $\mathrm{m}$ & 86 & 15 & 45 & 8 & 48 & 8 & 38 & 6 & 93 & 16 & 20 & 3 & 89 & 16 & 71 & 12 & 62 & 11 & 552 & 49 \\
\hline $\mathrm{h}$ & 53 & 9 & 39 & 6 & 16 & 2 & 39 & 6 & 94 & 16 & 12 & 2 & 189 & 33 & 55 & 9 & 72 & 12 & 569 & 50 \\
\hline
\end{tabular}

\subsubsection{Análisis de los factores sociales en la atenuación}

\section{a. Sexo}

Tabla 2. Uso de recursos atenuadores y el género

\begin{tabular}{|c|c|c|c|c|c|c|}
\hline & D & E & M. así & P & C & 0 \\
\hline H & .349 & .430 & .308 & .648 & .410 & .675 \\
\hline M & .656 & .572 & .698 & .348 & .593 & .320 \\
\hline
\end{tabular}




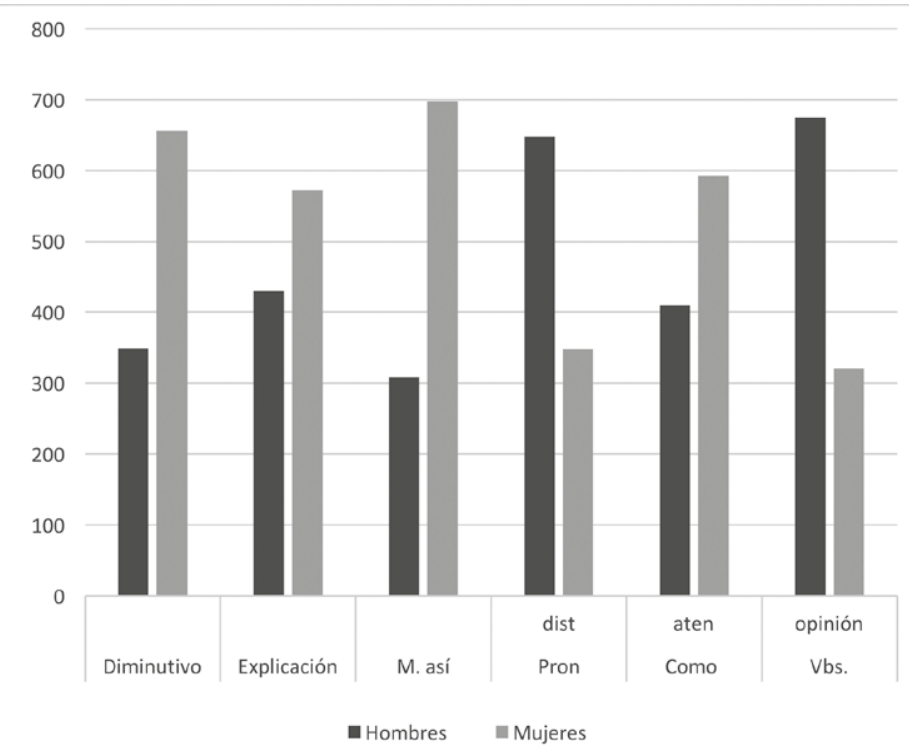

Figura 1. Recursos atenuadores según el sexo

El análisis binomial demuestra la clasificación de recursos atenuadores según el sexo (el Goldvarb excluyó la expresión de duda, los retardadores y el no saber): las mujeres prefieren atenuar con el diminutivo, la explicación, el marcador "así" y el "como" atenuador. Mientras los hombres, por su parte, optan por los pronombres de distanciamiento y los verbos de opinión.

\section{b. Edad}

Tabla 3. Uso de recursos atenuadores según la edad

\begin{tabular}{|c|c|c|c|l|l|l|l|}
\hline & Diminutivo & Explicación & Ex. Duda & $\begin{array}{l}\text { M. } \\
\text { retardadores }\end{array}$ & No saber & $\begin{array}{l}\text { Pron. } \\
\text { Dist. }\end{array}$ & $\begin{array}{l}\text { Vbs. } \\
\text { opinión }\end{array}$ \\
\hline $20-34$ & .368 & .432 & .681 & .62 & .630 & .357 & .683 \\
\hline $35-54$ & .607 & .514 & .385 & .50 & .536 & .600 & .334 \\
\hline $55-$ & .718 & .676 & .179 & .17 & .138 & .754 & .231 \\
\hline
\end{tabular}




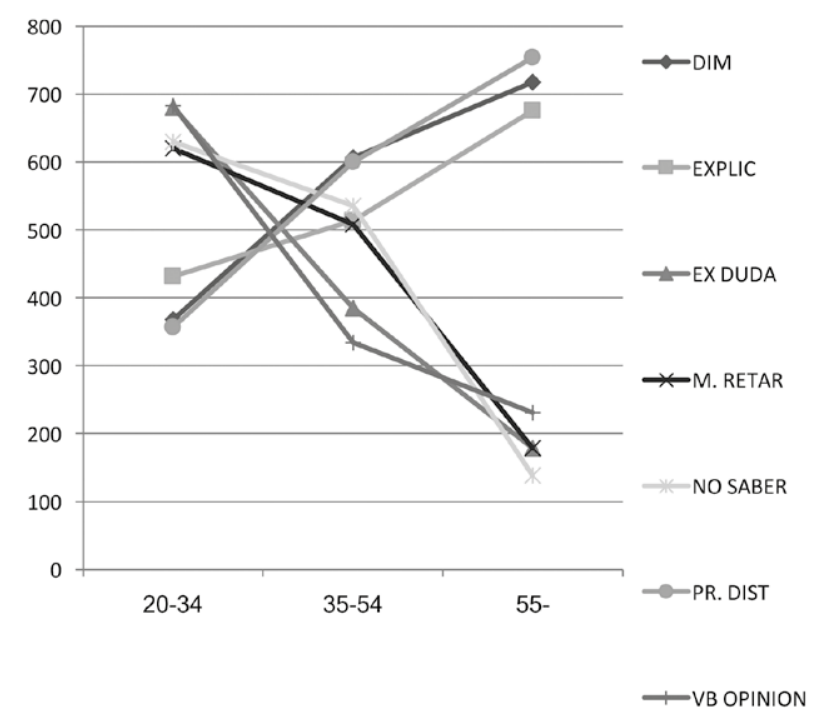

Figura 2. Atenuantes según la edad: resultados inferenciales.

El Goldvarb excluyó el marcador "así", el "como" atenuador. De los datos, se puede plantear que el grupo entre los 20 y los 34 años tiende a usar los atenuadores de expresión de duda, los marcadores retardadores, el "no saber" y los verbos de opinión. La segunda generación, de los 35 a los 54 años, prefieren los recursos del diminutivo, la explicación, la expresión de duda, los marcadores retardadores, el "no saber" y los pronombres de distanciamiento. Los mayores de 55 años, por su parte, usan con alta probabilidad los diminutivos y los pronombres de distanciamiento; también presentan coeficientes altos para el mecanismo de la explicación.

Si se comparan los grupos etarios, se encuentran semejanzas y diferencias: las dos primeras generaciones coinciden en el uso de las expresiones de duda, los marcadores retardadores y el uso del "no saber"; en esto se diferencian de la tercera generación. Pero esta, a su vez, se asemeja a la segunda en el uso de: diminutivos, explicaciones y pronombres de distanciamiento, como estrategias atenuadoras.

Finalmente, es de resaltar que en lo que concierne a los verbos de opinión como estrategia atenuadora, solo los jóvenes entre los 20 y 34 años presentaron índices altos. Las otras dos generaciones, $2^{\mathrm{a}}$ y $3^{\mathrm{a}}$, poseen coeficientes bajos. Parece ser entonces una estrategia mitigadora identitaria de la primera generación.

En cuanto al mayor uso de algunos atenuadores por lo jóvenes, es necesario aclarar que se trata de un registro semi-informal. Ahora bien, también hay que tener en cuenta 
los participantes de la conversación semidirigida que pueden plantear una asimetría en el estatus y rol social. No obstante, es importante el resultado porque nos muestra que los jóvenes barranquilleros, cuando se encuentran en una situación tendiente a la formalidad, utilizan los atenuadores en alta proporción, y esto forma parte tanto de su competencia y actuación comunicativas.

\section{c. Nivel de instrucción}

El análisis frecuencial arrojó lo siguiente:

Tabla 4. Uso de recursos atenuadores según el nivel de instrucción: análisis frecuencial

\begin{tabular}{|c|c|c|c|c|c|c|c|c|c|c|c|c|c|c|c|c|c|c|c|c|}
\hline & \multicolumn{2}{|c|}{ d } & \multicolumn{2}{|c|}{ e } & \multicolumn{2}{|c|}{ a } & \multicolumn{2}{|c|}{$\mathrm{X}$} & \multicolumn{2}{|c|}{$\mathrm{m}$} & \multicolumn{2}{|c|}{$\mathrm{n}$} & \multicolumn{2}{|c|}{$\mathrm{p}$} & \multicolumn{2}{|c|}{ t } & \multicolumn{2}{|c|}{0} & \multicolumn{2}{|c|}{ TOTAL } \\
\hline & $\mathbf{N}$ & $\%$ & $\mathbf{N}$ & $\%$ & $\mathbf{N}$ & $\%$ & $\mathbf{N}$ & $\%$ & $\mathbf{N}$ & $\%$ & $\mathbf{N}$ & $\%$ & $\mathbf{N}$ & $\%$ & $\mathbf{N}$ & $\%$ & $\mathbf{N}$ & $\%$ & $\mathbf{N}$ & $\%$ \\
\hline $\mathrm{b}$ & 30 & 17 & 11 & 6 & 2 & 1 & 5 & 2 & 37 & 21 & 3 & 1 & 71 & 41 & 4 & 2 & 9 & 5 & 172 & 15 \\
\hline $\mathrm{m}$ & 51 & 10 & 45 & 9 & 34 & 6 & 33 & 6 & 52 & 10 & 10 & 2 & 103 & 20 & 61 & 12 & 105 & 21 & 494 & 44 \\
\hline $\mathrm{a}$ & 58 & 12 & 28 & 6 & 28 & 6 & 39 & 8 & 98 & 21 & 19 & 4 & 104 & 22 & 61 & 13 & 20 & 4 & 455 & 40 \\
\hline
\end{tabular}

Los datos frecuenciales demuestran que el nivel de instrucción bajo es el que menos atenuadores usa; los niveles medio y alto los utilizan en proporciones análogas.

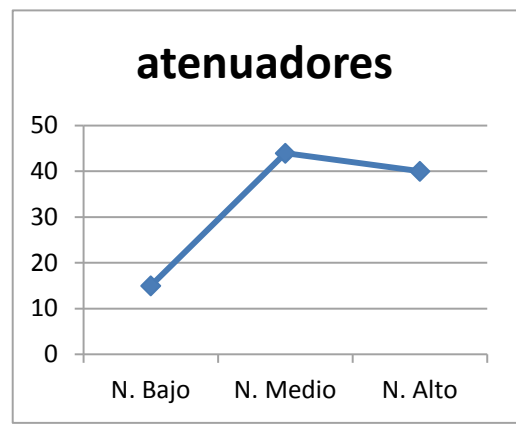

Figura 3. Atenuantes según el nivel de instrucción: resultado frecuencial.

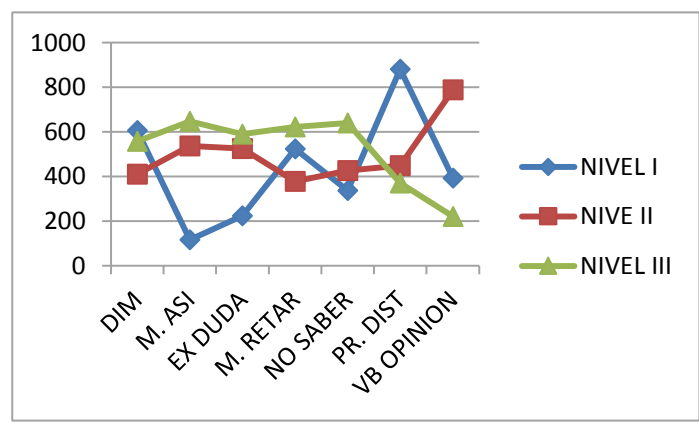

Figura 4. Atenuantes y el nivel de instrucción: resultado inferencial análisis inferencial.

Véase la distribución según los tipos de atenuadores a nivel inferencial:

Tabla 5. Uso de recursos atenuadores según nivel de instrucción: análisis inferencial

\begin{tabular}{|c|c|c|c|l|l|l|l|l|}
\hline & Dim. & M. así & $\begin{array}{l}\text { E x } \\
\text { duda }\end{array}$ & $\begin{array}{l}\text { M. } \\
\text { Ret. }\end{array}$ & $\begin{array}{l}\text { N o o } \\
\text { sab. }\end{array}$ & $\begin{array}{l}\text { P. } \\
\text { Dis. }\end{array}$ & $\begin{array}{l}\text { Co. } \\
\text { At. }\end{array}$ & $\begin{array}{l}\text { Vbs. } \\
\text { Op. }\end{array}$ \\
\hline N. I & .606 & .116 & .224 & .524 & .337 & .881 & .139 & .393 \\
\hline N. II & .411 & .537 & .525 & .378 & .427 & .449 & .539 & .788 \\
\hline N.III & .557 & .647 & .590 & .623 & .640 & .370 & .627 & .221 \\
\hline
\end{tabular}


El nivel de instrucción también demostró ser un factor social influyente en la selección de los recursos de atenuación por parte de los hablantes. Se puede apreciar resultados congruentes con la escala social como, por ejemplo, en los atenuadores correspondientes a: el marcador "así", las expresiones de duda, el uso del "no saber", los pronombres de distanciamiento y el "como" atenuador (El Goldvarb excluyó la explicación). En el primer recurso se aprecia que, conforme aumenta el nivel de instrucción, se incrementa el uso del "así" atenuador, el cual es probable en los niveles II y III; pero de baja probabilidad en el nivel I. Esto mismo ocurre con las expresiones de duda y el "como" atenuador. En lo que respecta al "no saber", se observa también un incremento conforme aumenta la instrucción en los hablantes, pero es significativo con probabilidad favorable para el nivel III. En lo que concierne a los pronombres de distanciamiento, conforme disminuye el nivel de instrucción, aumenta la probabilidad de uso; pero solo es significativo y altamente probable en el nivel I.

Sin embargo, no todos los resultados son congruentes con la escala sociocultural; también se encuentran resultados dispares en los recursos: utilización del diminutivo, probable en los niveles I y III, siendo de mayor preferencia en el primero; pero no en el II; en los marcadores retardadores, se da el mismo patrón. En estos recursos se asemejan entonces, los niveles I y III.

En cuanto al grupo II, vale la pena anotar que solo en él se halló un coeficiente bastante alto; lo cual indica que es un recurso atenuador identitario de este grupo.

Para finalizar, este análisis demuestra que ciertamente existe una distribución social de los recursos atenuadores; más allá de las funciones pragmáticas que puedan cumplir, se encuentra que no todos los grupos los usan de la misma manera en la comunidad de habla barranquillera. No obstante, también es importante mencionar la variación en la distribución social de los recursos; pues, si bien unos son sensibles a ciertos factores sociales, otros no se comportan de la misma manera. Por ejemplo, el factor sexo no presentó ninguna incidencia en la distribución social de los marcadores retardadores y el "no saber". Esto quiere decir, que tanto hombres como mujeres los emplean en proporciones análogas y no se diferencian. El factor edad resultó no significativo para el "como" atenuador. Ciertamente, los grupos etarios no se distinguen entre sí en este recurso, sino que lo utilizan con la misma probabilidad. Y el factor nivel de instrucción, solo el recurso de las explicaciones, resultó no significativo.

Comparando los tres factores sociales, el que más incide en la distribución de los recursos de atenuación dentro de la comunidad de habla barranquillera es el nivel de instrucción. Esto quiere decir que la educación formal desempeña un papel importante en la disponibilidad y uso de los atenuadores, creando variabilidad. 


\section{d. Variable género discursivo}

Analizadas las variables independientes externas, se estudia a continuación el factor interno: la incidencia de los géneros discursivos en el uso de las marcas atenuadoras en la comunidad de habla Barranquillera. Como se observa en la siguiente tabla, en el género argumentativo es más frecuente la utilización de los atenuadores: 31\% (narrativo), 68\% (argumentativo). Los barranquilleros atenúan más en este último³ ${ }^{3}$.

Tabla 6. Uso de recursos atenuadores según género discursivo

\begin{tabular}{|c|c|c|c|c|c|c|c|c|c|c|c|c|c|c|c|c|c|c|c|c|}
\hline & & 1 & e & & $a$ & & $Y$ & & $N$ & & 1 & & $\mathrm{p}$ & & ( & & & & T0' & \\
\hline & $\mathbf{N}$ & $\%$ & $\mathbf{N}$ & $\%$ & $\mathbf{N}$ & $\%$ & $\mathbf{N}$ & $\%$ & $\mathbf{N}$ & $\%$ & $\mathbf{N}$ & $\%$ & $\mathbf{N}$ & $\%$ & $\mathbf{N}$ & $\%$ & $\mathbf{N}$ & $\%$ & $\mathbf{N}$ & $\%$ \\
\hline $\mathrm{n}$ & 90 & 20 & 46 & 10 & 43 & 9 & 44 & 10 & 30 & 6 & 54 & 12 & 60 & 13 & 42 & 9 & 29 & 6 & 438 & 31 \\
\hline$a$ & 195 & 20 & 134 & 14 & 126 & 13 & 82 & 8 & 52 & 5 & 84 & 8 & 135 & 14 & 89 & 9 & 59 & 6 & 956 & 68 \\
\hline
\end{tabular}

Como se puede apreciar, cuando los barranquilleros argumentan, prefieren utilizar los pronombres de distanciamiento, los marcadores retardadores y los diminutivos, respectivamente; mientras que cuando narran, optan por los pronombres de distanciamiento, los marcadores retardadores y las explicaciones. Nótese que, a pesar de la coincidencia en cuanto a la selección de las marcas atenuadoras, se observa una diferencia significativa en la frecuencia de uso, siendo más usadas en el discurso argumentativo.

\section{e. El interlocutor}

El tipo de interlocutor también posee una incidencia en la variación del uso de los atenuantes en Barranquilla. Se analizó el sexo del hablante (entrevistado) y del interlocutor (entrevistador), para observar si la frecuencia se modificaba; véase el siguiente resultado:

Tabla 7. Atenuantes y tipo de interlocutor

\begin{tabular}{|l|l|l|l|}
\hline \multicolumn{2}{|l|}{ Atenuantes } & Hablante & Interlocutor \\
\hline $\mathrm{N}$ & $\%$ & & \\
\hline 513 & 37.6 & Hombre & Hombre \\
\hline 168 & 12.3 & Hombre & Mujer \\
\hline 440 & 32.6 & Mujer & Mujer \\
\hline 243 & $\mathbf{1 7 . 8}$ & Mujer & Hombre \\
\hline
\end{tabular}

Tabla 8. Atenuantes y tipo de interlocutor: pares del mismo sexo

\begin{tabular}{|l|c|l|c|}
\hline & $\begin{array}{l}\text { Debilitadores } \\
\text { Atenuadores } \\
\text { Brown Tzeltal }\end{array}$ & $\begin{array}{l}\text { Debilitadores } \\
\text { Atenuadores } \\
\text { Rodríguez. } \\
\text { Barranquilla }\end{array}$ & $\begin{array}{l}\text { Total de } \\
\text { partículas }\end{array}$ \\
\hline $\begin{array}{l}\text { Recurso } \\
\text { en pares } \\
\text { femeninos }\end{array}$ & 34.1 & 32.6 & 59.3 \\
\hline
\end{tabular}

3 En este análisis la codificación para el Goldvarb varió así: p: pronombre de distanciamiento; d: diminutivo; c: cómo atenuado; x: expresiones de duda; f: modificación morfológica externa. k: explicación; r: marcadores retardadores; y: verbos de opinión; z. 
La tabla 7 muestra que cuando el hablante y el oyente son del mismo sexo, los atenuantes en aquel, tienden a aumentar; cuando son de diferentes sexos, estos disminuyen. Ahora bien, cuando el hablante es mujer y el oyente es hombre, la atenuación tiende a aumentar levemente, con respecto al caso cuando el hablante es hombre y el interlocutor es mujer. Este resultado es comparable con el encontrado por Brown (citado por Coates, 2009) quien estudió el promedio de partículas fortificadoras o debilitadoras, en 100 actos lingüísticos en pares formados por personas del mismo sexo en tzeltal, lengua maya en la que se encuentran unas partículas que funcionan como adverbios y modifican la fuerza del acto lingüístico; véanse a continuación los datos:

Estos resultados son análogos a los que se hallaron en Barranquilla. La coincidencia de sexos en las mujeres aumenta el uso de atenuadores. En pares de género mixto, también se puede comparar la comunidad de habla barranquillera con la tzeltal; véanse los siguientes datos:

Tabla 9. Atenuantes y tipo de interlocutor: pares de género mixto

\begin{tabular}{|l|l|c|c|}
\hline & $\begin{array}{l}\text { Debilitadores } \\
\text { Atenuadores } \\
\text { Rodríguez. } \\
\text { Barranquilla }\end{array}$ & $\begin{array}{l}\text { Debilitadores } \\
\text { Brown Tzeltal }\end{array}$ & $\begin{array}{l}\text { Total de } \\
\text { partículas }\end{array}$ \\
\hline Recurso en mujeres que se dirigen a hombres & 17.8 & 24.4 & 59.3 \\
\hline Recurso en hombres que se dirigen a mujeres & 12.3 & 33.1 & 57.2 \\
\hline
\end{tabular}

Los resultados son distintos en los pares mixtos; en Barranquilla, los atenuadores aumentan cuando una mujer se dirige a un hombre; en Tzeltal, disminuyen.

La incidencia del tipo de interlocutor en la frecuencia de uso de las marcas de atenuación, ya había sido anotada por Albelda Marco (2006), quien plantea que: "El número de atenuantes y su diverso grado de mitigación en una situación comunicativa reflejará la relación de más proximidad o distancia, de más igualdad o desigualdad social y funcional entre los interlocutores" (p. 99). Agrega la autora que esta variabilidad también se debe a los distintos patrones culturales, además de la situación comunicativa; y que dicha diferenciación debe interpretarse como un contínuum gradual.

\section{Conclusiones}

Los atenuantes en Barranquilla en su uso y frecuencia, covarían con los factores sociales sexo, edad y nivel de instrucción; con el género discursivo y con el factor pragmático, tipo de interlocutor. Las variables externas más significativas fueron edad y nivel edu- 
cativo; conforme aumenta la edad, disminuyen los atenuantes; y conforme se incrementa el grado de instrucción, aumentan estos elementos en el habla barranquillera. En cuanto al género discursivo, es el argumentativo el que presenta más marcas atenuadoras; mientras que, en el narrativo, si bien disminuyen dichas marcas, aparecen cuando el hablante incrusta estructuras argumentativas o descriptivas en su relato, que amenazan la imagen del interlocutor.

Finalmente, el tipo de interlocutor también influye en el uso de los atenuadores; cuando el hablante y el interlocutor coinciden en el sexo, estos son más frecuentes.

\section{Referencias}

Albelda Marco, M. (2004). Cortesía en diferentes situaciones comunicativas, la conversación coloquial y la entrevista sociológica semiformal. En D. Bravo, \& A. Briz (eds.), Pragmática sociocultural: Estudios sobre cortesía en español. Barcelona: Ariel.

Albelda Marco, M. (2005). Discordancia entre atenuación/cortesía e intensificación/descortesía en conversaciones coloquiales. En: J. L. Blas, M. Casanova, \& M. Velando (eds.), Discurso y Sociedad. Contribuciones al estudio de la lengua en contexto social.

Albelda Marco, M. (2006). Atenuantes en Chile y en España: distancia o acercamiento. En: A. Briz et al. (2008), Cortesía y conversación: de lo escrito a lo oral. III Coloquio Internacional del Programa EDICE. España: Universitat de València.

Albelda, M., \& Briz, A. (2013). Una propuesta teórica y metodológica para el análisis de la atenuación lingüística en español y portugués. La base de un proyecto en común (ES. POR. ATENUACIÓN). Onomázein, 28, 288-319. D0I: 10.7764/onomazein.28.21.

Bravo, D., \& Briz, A. (2004). Atenuación y cortesía verbal en la conversación coloquial. Pragmática sociocultural: análisis del discurso de cortesía en español. Barcelona: Ariel.

Bravo, D., \& Briz. A. (eds.) (2004). La cortesía como búsqueda del equilibrio de la imagen social. Pragmática sociocultural: análisis del discurso de cortesía en español. Barcelona: Ariel.

Bravo, D., \& Briz, A. (eds.). (2004). Cortesía verbal codificada y cortesía verbal interpretada. Pragmática sociocultural: estudios sobre el discurso de cortesía en español. España: Ariel.

Ballesteros, F. (2002). Mecanismos de atenuación en español e inglés. Implicaciones pragmáticas en la cortesía. Círculo de lingüística aplicada a la comunicación, 11. Recuperado de http://www.ucm.es/info/circulo/no11/ballesteros.htm.

Briz, A. (2001). El español coloquial en la conversación. Esbozo de pragmagramática (2ª . Ed.). Barcelona, España: Ariel. 
Briz, A. (2002). El español coloquial en la clase de E/LE. Un recorrido a través de los textos. Madrid, SGEL: Sociedad general española de librería, S.A.

Briz A. (2002). La estrategia atenuadora en la conversación cotidiana española. Actas del Primer Coloquio del Programa Edice. Suecia: Universidad de Estocolmo.

Briz, A. (2003). La estrategia atenuadora en la conversación cotidiana española. En: D. Bravo (ed.) Actas del Primer Coloquio del programa EDICE. La perspectiva no etnocentrista de la cortesía: Identidad sociocultural de las comunidades hispanohablantes (pp. 17-46). Estocolmo: Universidad de Estocolmo.

Brown y Levinson. (1987). La cortesía. Cambridge: Cambridge University Press.

Caffi, C. (1999). On mitigation. Journal of Pragmatics, 3.

Cisneros Estupiñán, M. (2007). Atenuación de los mandatos y las peticiones en la ex provincia de Obando. Lenguaje, 35(1), 29-46.

Coates, J. (2009). Mujeres, hombres y lenguaje. Un acercamiento sociolingüístico a las diferencias de géneros. México: Fondo de Cultura Económica.

Coutin, I. (2010). Los fenómenos de intensificación y atenuación en el discurso de los vendedores en los buses de transporte público de Medellín, Antioquia. Medellín: Fundación el libro total.

Charaudeau, P. (1992). Las emociones como efectos de discurso. Revista Versión, 26, 97-118.

Félix-Brasdefer, J.C. (2004). La mitigación en el discurso oral de mexicanos y aprendices de español como lengua extranjera. En: Bravo y A. Briz (2004), Pragmática sociocultural: Estudios sobre cortesía en español. Barcelona: Ariel.

Fraser, B. (1999). Mitigation. Journal of Pragmatics, 31.

Garrido Rodríguez, M. (2006). Gramaticalización y marcadores del discurso: los contraargumentativos. Estudios Humanísticos. Filología, 28, 9-26.

Gumperz (1971). Comunidad lingüística y comunidad de habla. La traducción entre lenguas en contacto: catalán y español.

Haverkate, H. (1994). La cortesía verbal. Estudio pragmalingüístico. Madrid: Gredos.

Hidalgo Navarro, A. (2004). El corpus de conversación coloquial del Grupo Val.Es.Co. (Valencia, Español Coloquial). Revista Actas de las primeras jornadas sobre lengua y sociedad, 219216. 
Kerbrat Y Orecchioni. (1996). Concepto de cortesía. Discurso y Sociedad: contribuciones al estudio de la lengua en contexto social. Universitat Jaume.

Labov, W. (1966). Diversidad lingüística. Antropología lingüistica. Cambridge: University of Cambridge.

Labov, W. (2009). Comunidad lingüística y comunidad de babla. La traducción entre lenguas en contacto: catalán y español.

Lakoff, J. (1973). La Cortesía Lingüística en el discurso publicitario. Valéncia: Universitat de Valéncia.

Martínez Solís, M. (2002). Estrategias de la lectura y escritura de textos. Perspectivas teóricas y talleres. Cali: Artes gráficas de la facultad de humanidades de la universidad del Valle.

Martinovski, B. (2000). The Role of Repetitions and Reformulations in Court Proceedings: A Comparison between Sweden and Bulgaria. (Ph.D. Thesis). Goteborg University Press, Sweden.

Moreno Fernández, F. (1990). Metodología sociolingüística. Madrid: Gredos.

Moreno Fernández, F. (1998). Principios de sociolingüística y sociología del lenguaje. Barcelona: Ariel.

Ortega Salamanca, F., \& Vargas Cortés, B. (2017). Aproximación sociopragmática a las estrategias conversacionales de los adolescentes. Cuadernos de Lingüística Hispánica, (29), 83-103. doi: https://doi.org/10.19053/0121053X.n29.2017.5849

Puga Larraín, J. (1997). La atenuación en el castellano de Chile: un estudio pragmalingüístico. Valencia: Universitat de Valéncia.

Real Academia Española. (1970). Diccionario de la Lengua Española. (29a ed.). Madrid: Espasa Calpe.

Rodríguez Cadena, Y. (2008). El Habla de Barranquilla: materiales para su estudio. Preseea Barranquilla Tomo I. Barranquilla: Universidad del Atlántico.

Rodríguez Cadena, Y. (2010). Estudio sociolingüístico de los atenuantes en Barranquilla. Ponencia en EDICE, Barranquilla, Colombia.

Romaine. (1982). Comunidad lingüística y comunidad de habla. La traducción entre lenguas en contacto: catalán y español.

Silva Corvalán, C. (2001). Sociolingüística y Pragmática del español. EE.UU.: Georgetown University Press. 
Torres Fontalvo, Y. (2014). La atenuación en el habla de Barranquilla: un estudio sociolingüístico (tesis de Maestría). Universidad del Atlántico, Barranquilla, Colombia.

Van Eemeren, F.H., \& Grootendorst, R. (2004). A Systematic. Theory of Argumentation: The PragmaDialected. Approach. United Kingdom: Cambridge University Press.

Weinreich, U. (1966). On the Semantic Structure of English. En: A. González Salinas. XVII Congreso internacional de Lingüística y Filología de América latina (ALFAL 2014), Paraiba, Brasil.

Zadeh, L. (1965). Fuzzy Sets, Information and control, 8, 338-353. 